\title{
ON AN ELLIPTIC BOUNDARY VALUE PROBLEM NOT IN DIVERGENCE FORM
}

\author{
NGUYÊN PHUONG CÁC
}

\begin{abstract}
Let $G$ be a bounded domain in $R^{n}(n \geqslant 2)$ with smooth boundary $\partial G$. We consider the boundary value problem $M u-c u=f$ on $G, u=0$ on $\partial G$, where $M$ is an elliptic differential operator not in divergence form. We discuss the characterization of the first eigenvalue $\lambda_{0}$ of $M$ and the solvability of the boundary value problem in terms of the relationship between $c(\cdot)$ and $\lambda_{0}$.
\end{abstract}

I. Introduction. Let $G$ be a bounded domain in $R^{n}(n \geqslant 2)$ with smooth boundary $\partial G$. In this paper we discuss the boundary value problem (BVP)

$$
\begin{gathered}
M u-c(x) u=f \text { in } G, \\
u=0 \text { on } \partial G
\end{gathered}
$$

where $f \in L^{p}(G)$ with $p>1, c \in L^{s}(G)$ for some $s>1$ to be specified later, and $M$ is the differential operator

$$
M=-a_{i j} \frac{\partial^{2}}{\partial x_{i} \partial x_{j}}+b_{i} \frac{\partial}{\partial x_{i}}
$$

in which the usual summation convention is used: if an index is repeated then the summation over it from 1 to $n$ is implied. We assume throughout the paper that $a_{i j} \in C(\bar{G}), b_{i} \in L^{\infty}(G)$ for $i, j=1,2, \ldots, n$, and the operator $M$ is uniformly elliptic, i.e., there is a constant $\mu>0$ such that in $\bar{G}$,

$$
a_{i j} \zeta_{i} \zeta_{j}>\mu|\zeta|^{2} \quad \forall \zeta \in R^{n}, \zeta \neq 0 .
$$

For ane sake of simplicity we consider only real functions. Let

$$
\begin{aligned}
& \rho=\sup \{\lambda \geqslant 0: M u-\lambda u=1 \text { has a nonnegative solution } \\
& \left.\qquad u \in W^{2, q}(G) \cap W_{0}^{1, q}(G) \text { for any finite } q>1\right\} .
\end{aligned}
$$

Theorem 1 below shows that if the operator $M$ has a smallest eigenvalue then this eigenvalue is equal to $\rho$. This result is embodied in Part 2, Proposition III.2 of [5]. The proof in [5] appears to make essential use of a formula of Ito in ergodic theory. We provide a proof based only on a version of the generalized maximum principle due to Serrin [6] and adapted to the Sobolev space $W^{2, p}(G)$. Theorem II shows that if the quantity $\rho$ in (3) is finite then the operator $M$ has a smallest eigenvalue. Theorem III proves the solvability of the boundary value problem (1), (2) when $c(\cdot)$

Received by the editors November 16, 1981.

1980 Mathematics Subject Classification. Primary 35J25; Secondary 47B05.

Key words and phrases. First eigenvalue, solvability of boundary value problem.

(C)1983 American Mathematical Society 0002-9939/82/0000-1091/\$02.25 
is bounded above away from the smallest eigenvalue of $M$. The corollary to Theorem III appears to be what we should naturally expect. It has already been stated as Part 1, Proposition III.2 in [5], but without a proof and, as far as we can see, it does not seem to follow trivially from any result in [5] either. Theorem $\mathrm{V}$ deals with the case when the spectrum of $M$ is empty. It seems to us that this eventuality was not considered in [5].

II. Main results. We first recall some results needed in the sequel.

LemMA I (MAXIMUM PRINCIPLE IN $W^{2, p}(G)$ ). Suppose that for $u \in W^{2, p}(G) \cap$ $W_{0}^{1, p}(G), h(x) \geqslant 0$ on $G$ we have $M u+h u \geqslant 0$ on $G$.

(i) If $h \in L^{s}(G)$ where $s=n / 2$ if $1<p<n / 2, s>n / 2$ if $p=n / 2$, and $s=p$ if $p>n / 2$, then $u \geqslant 0$ in $G$.

(ii) If $h \in L^{\infty}(G)$, then either $u \equiv 0$ or $u$ is essentially bounded from below by $a$ positive number on every compact subset of $G$.

Part (ii) is essentially Corollary I.1 of [5]. In the case $p>n$ it was proved earlier in [2, Theorem 2]. Part (i) can be found in [3, Theorem 2].

Lemma II (Generalized MAXIMUM PRINCIPLE OF Serrin [6]). Let $u \in W^{2 . p}(G) \cap$ $W_{0}^{1, p}(G)$ satisfy for some number $\lambda$ the inequality $M u-\lambda u \geqslant 0$ in $G$. If there exists a function $w \in W^{2, p}(G) \cap W_{0}^{1, p}(G)$ such that $M w-\lambda w \geqslant g(x), w>0$ in $G$ where $g(\cdot)$ is continuous and bounded on $G, g(x)>0$ for every $x \in G$. Then either $u \equiv 0$ or $u>0$ in $G$.

In [6] $u, w \in C^{2}(G) \cap C(\bar{G})$ and all coefficients of $M$ are assumed continuous. A careful reading of [6] indicates that, in our context, the purpose of this continuity requirement is to insure that if $M w(x)-\lambda w(x)>0$ for each $x \in G$ then $M x-\lambda w$ has a positive lower bound on each compact subset of $G$ but this is certainly true under our assumption. Then instead of using the maximum principle in $C^{2}(G)$ we use the maximum principle in $W^{2, p}(G)$.

If the operator $M-\lambda I$ considered as an operator on $L^{q}(G)(q>1$, finite) with domain $W^{2, q}(G) \cap W_{0}^{1, q}(G)$ has a continuous inverse on $L^{q}$ then we denote it by $R(\lambda, M)$. It is known [3, Theorem 2; 5, Theorem I.1 and Corollary I.1] that $R(0, M)$ exists and maps the positive cone of $L^{q}(G)$ into itself, i.e., it is a positive mapping. If the spectral radius of $R(0, M)=M^{-1}$ is $>0$ then we know from the Krein-Rutman theorem [4] that $M^{-1}$ has a largest eigenvalue with a nonnegative eigenfunction. It then follows that $M$ has a smallest (usually called first) eigenvalue $\lambda_{0}>0$ and the maximum principle in $W^{2 . q}(G)$ shows that there corresponds an eigenfunction $\phi$, $\phi>0$ in $G$.

It is well known that if the coefficients of $M$ are smoother than assumed here, namely if they belong to $C^{\beta}(\bar{G}), \beta \in(0,1)$, then this first eigenvalue exists (cf., e.g., [1, Theorem 4.3]).

THEOREM 1. Suppose that the first eigenvalue $\lambda_{0}$ of $M$ exists. Then $\lambda_{0}=\rho$ where $\rho$ is defined in (3). 
Proof. We first prove that $\rho \geqslant \lambda_{0}$. In fact, for any $\lambda<\lambda_{0}$, the equation

$$
M u-\lambda u=1
$$

has a unique solution $u \in W^{2, q}(G) \cap W_{0}^{1, q}(G)$ because $\lambda$ belongs to the resolvent set of $M$. We have

$$
M \phi-\lambda \phi=\left(\lambda_{0}-\lambda\right) \phi>0 .
$$

Since $\phi \in W^{2, q}(G)$, for sufficiently large $q, \phi$ is continuous and bounded on $G$. Using the generalized maximum principle (Lemma II), we deduce from (4), (5) that $u>0$ in $G$. Thus $\lambda$ belongs to the set on the right-hand side of (3) and $\rho \geqslant \lambda_{0}$.

We now prove that $\lambda_{0} \geqslant \rho$. Suppose by contradiction that $\lambda_{0}<\rho$. Then for $\varepsilon>0$ sufficiently small, $\lambda_{0}+\varepsilon<\rho$ and the equation

$$
M u-\left(\lambda_{0}+\varepsilon\right) u=1
$$

has a solution $u>0$ in $G$ by definition of $\rho$. On the other hand,

$$
M \phi-\left(\lambda_{0}+\varepsilon\right) \phi=-\varepsilon \phi<0 .
$$

Again, using Lemma II, we deduce from (6), (7) that $\phi \leqslant 0$. But this is not possible. Therefore $\lambda_{0}=\rho$. Q.E.D.

The referee has suggested an alternate proof for Theorem 1. It can be found at the end of the paper.

THEOREM II. If the number $\rho$ defined in (3) is finite, then the first eigenvalue $\lambda_{0}$ of $M$ exists (and $\left.\lambda_{0}=\rho\right)$.

Proof. It suffices to show that if $\rho$ is finite then $\rho$ is an eigenvalue of $M$. The other claims then follow from previous discussions. Suppose by contradiction that it is not. Then $\rho$ belongs to the resolvent set of $M$ and $R(\rho, M)$ exists. Then for $\varepsilon>0, \varepsilon$ sufficiently small, the interval $(\rho-\varepsilon, \rho+\varepsilon)$ is contained in the resolvent set of $M$ and $\rho-\varepsilon>0$. We claim that for $\lambda \in(\rho-\varepsilon, \rho) R(\lambda, M)$ is a positive mapping in $L^{q}(G)$. In fact, for $f \in L^{q}(G), f \geqslant 0$ in $G$, let $w=R(\lambda, M) f$. Then $w \in W^{2, q}(G) \cap$ $W_{0}^{1, q}(G)$ and $M w-\lambda w=f \geqslant 0$. Since $\lambda<\rho$ there is $u \in W^{2, q}(G) \cap W_{0}^{1, q}(G)$, $u \geqslant 0$, in $G$ with $M u-\lambda u=1>0$. It follows from Lemma I that actually $u>0$ in $G$. Using Lemma II we deduce that $w \geqslant 0$ in $G$. Then by letting $\lambda \uparrow \rho$, it is not difficult to see that $R(\rho, M)$ is a positive mapping in $L^{q}(G)$. On the other hand, for $\lambda \in(\rho, \rho+\varepsilon)$ we have

$$
R(\lambda, M)=R(\rho, M)\left\{I+\sum_{n=1}^{\infty}(\lambda-\rho)^{n} R(\rho, M)^{n}\right\} .
$$

Thus $R(\lambda, M)$ is a positive mapping in $L^{q}(G)$ and there is a unique $u \in W^{2, q}(G) \cap$ $W_{0}^{1, q}(G), u>0$, in $G$ such that $M u-\lambda u=1$. But this violates the definition of $\rho$ in (3) because $\lambda>\rho$. Q.E.D.

THEOREM III. Suppose that the spectrum of $M$ is not empty and $c \in L^{s}(G)$ with $s=n / 2$ if $1<p<n / 2, s>n / 2$ if $p=n / 2$, and $s=p$ if $p>n / 2$. Suppose also that there exists $\alpha>0$ such that $c(x) \leqslant \lambda_{0}-\alpha$ where $\lambda_{0}$ is the first eigenvalue of $M$. Then 
for every $f \in L^{p}(G), f \geqslant 0$ in $G$ the $B V P(1)$, (2) has a unique solution $u \in W^{2, q}(G)$ $\cap W_{0}^{1, p}(G), u \geqslant 0$ in $G$ and there is a constant $K$ independent of $f$ such that

$$
\|u\|_{W^{2, p}} \leqslant K\|f\|_{L^{p}} .
$$

Proof. Let $\lambda=\lambda_{0}-\alpha$. From Theorem I it follows that there is a unique $w \in W^{2, q}(G) \cap W_{0}^{1, q}(G)$ for any finite $q>1, w>0$ in $G$ such that

$$
M w-\lambda w=1 \text {. }
$$

Since $\lambda$ is in the resolvent set of $M$, there is $\hat{u} \in W^{2, p}(G) \cap W_{0}^{1 . p}(G)$ such that

$$
M \hat{u}-\lambda \hat{u}=f \geqslant 0 \text {. }
$$

Using Lemma II we deduce from (9) and (10) that $\hat{u} \geqslant 0$ in $G$. We have

$$
M \hat{u}-c \hat{u}=f+[\lambda-c] \hat{u} \geqslant f .
$$

Thus $\hat{u}$ is an upper solution of the $\operatorname{BVP}(1),(2)$. Now let $c^{-}(x)=\max \{0,-c(x)\}$. It is known $[3,5]$ that there is a unique $\underline{u} \in W^{2 . p}(G) \cap W_{0}^{1, p(G)}, \underline{u} \geqslant 0$ in $G$ such that $M \underline{u}+c^{-} \underline{u}=f$. Then

$$
M \underline{u}-c \underline{u}=f-\left(c^{-}+c\right) \underline{u} \leqslant f .
$$

Thus $\underline{u}$ is a lower solution of the BVP (1), (2). We claim that $\hat{u} \geqslant \underline{u}$ in $G$. In fact, because $\lambda+c^{-}(x) \geqslant 0$ in $G$,

$$
M(\hat{u}-\underline{u})-\lambda(\hat{u}-\underline{u})=\left(\lambda+c^{-}\right) \underline{u} \geqslant 0 .
$$

Using Lemma II, we deduce from this and (9) that $\hat{u}-\underline{u} \geqslant 0$ in $G$. We now use the more or less standard method to show that a BVP which has an upper solution $\hat{u}$ and a lower solution $\underline{u}$ with $\hat{u} \geqslant \underline{u}$ has a solution. We sketch it. Consider the iteration scheme

$$
M u_{n}+c^{-} u_{n}=f+\left(c+c^{-}\right) u_{n-1} \quad \text { in } G, \quad u_{n}=0 \quad \text { on } \partial G
$$

with $u_{0}=\underline{u}$. It is not difficult to see that

$$
0 \leqslant \underline{u} \leqslant u_{1} \leqslant u_{2} \leqslant \cdots \leqslant u_{n} \leqslant \cdots \leqslant \hat{u} \quad \text { in } G .
$$

It is known [3, Theorem 2] that the mapping $M+c^{-}(x) I$ from $W^{2 . p}(G) \cap W_{0}^{1 . p}(G)$ to $L^{p}(G)$ is continuous, one-to-one and onto. Therefore it is well known that it has a continuous inverse. Hence, since $0 \leqslant c+c^{-} \leqslant \lambda_{0}-\alpha$, it follows from (11) that the sequence $\left\{u_{n}\right\}$ is bounded in $W^{2, p}(G)$. Consequently we can extract from it a subsequence converging weakly in $W^{2, p}(G) \cap W_{0}^{1, p}(G)$, strongly in $L^{p}(G)$ and a.e. on $G$ to a solution $u, u \geqslant 0$ on $G$, of the BVP (1), (2). Since $\hat{u}$ and $\underline{u}$ satisfy estimates similar to (8), it is not difficult to see from (11) that for the solution $u$ just obtained, (8) is valid. The uniqueness claimed is an immediate consequence of the following

THEOREM IV. Suppose that the function $c$ is as in Theorem III. If $u \in W^{2, p}(G) \cap$ $W_{0}^{1, p}(G)$ is such that $M u-c u=0$ in $G$, then $u \equiv 0$ in $G$.

Proof. Let

$$
G^{+}=\{x: x \in G, u(x)>0\}, \quad G^{-}=\{x: x \in G, u(x)<0\} .
$$

On $G^{+}$we have

$$
0=M u-c u \geqslant M u-\left(\lambda_{0}-\alpha\right) u \text {. }
$$


On the other hand, as has been pointed out in the proof of Theorem III, there is a $w \in W^{2, p}(G) \cap W_{0}^{1, p}(G), w>0$, in $G$ such that

$$
M w-\left(\lambda_{0}-\alpha\right) w=1 \text {. }
$$

Using Lemma II we deduce that $u \leqslant 0$ on $G^{+}$. Thus $u=0$ on $G^{+}$. Strictly speaking, the generalized maximum principle of Serrin (Lemma II) requires some smoothness for boundary $\partial G^{+}$. However, a careful reading of [6] indicates that the purpose of this smoothness requirement is to make possible the construction of a function positive on $\partial G^{+}$that would compensate for the possible vanishing of the referee function on that boundary. In our case, the referee function $w$ is positive on $\partial G^{+}-\partial G$ so that no smoothness of $\partial G^{+}-\partial G$ is necessary, whereas $\partial G$ is already assumed to be smooth. Similarly, on $G^{-}$we have

$$
0=M u-c u \leqslant M u-\left(\lambda_{0}-\alpha\right) u,
$$

and we deduce that $u \geqslant 0$ on $G^{-}$. Thus $u=0$ on $G^{-}$. Q.E.D.

Corollary to Theorem III. Let the function $c$ be as in Theorem III. Then for every $f \in L^{p}(G)$ the $B V P(1),(2)$ has a unique solution $u \in W^{2, p}(G) \cap W_{0}^{1, p}(G)$ and estimate (8) is valid.

Proof. The existence follows from Theorem III by decomposing $f$ into its positive and negative parts. The uniqueness follows from Theorem IV. The mapping $M-c I$ from $W^{2, p}(G) \cap W_{0}^{1, p}(G)$ to $L^{p}(G)$ is then continuous, one-to-one and onto. Hence it has a continuous inverse and we have (8).

THEOREM V. Suppose that the spectrum of the operator $M$ is empty and $c \in L^{s}(G)$ with $s$ as in Theorem III. Suppose also that $c(\cdot)$ is bounded from above. Then for every $f \in L^{p}(G)$ the $B V P$ (1), (2) has a unique solution $u \in W^{2, p}(G) \cap W_{0}^{1, p}(G)$ and estimate (8) is valid.

Proof. Let $\lambda$ be a number with $c(x) \leqslant \lambda$ on $G$. Since the spectrum of $M$ is empty, by Theorem II the quantity $\rho$ defined in (3) is infinite. Thus there is $w \in W^{2, p}(G) \cap$ $W_{0}^{1, p}(G), w>0$, on $G$ such that $M w-\lambda w=1$. The proof can then be completed exactly as for Theorem III and its corollary. Q.E.D.

The author wishes to thank an anonymous referee for suggesting the following alternate proof for Theorem I. It does not rely on the generalized maximum principle of Serrin.

Alternate proof of Theorem I. As stated in [7, Lemma 1], using Bony's maximum principle it can be shown that if $u \in W^{2, q}(G), q>n$, and for some $\gamma>0$, $M u+\gamma u \geqslant 0, u=0$ on $\partial G$ and $u \neq 0$, then $u>0$ in $G$ and $\partial u / \partial \nu<0$ on $\partial G$, where $\nu$ is the normal unit vector directed outward.

We first show that if $\tau<\lambda_{0}$ then the BVP

$$
M u-\tau u=1 \quad \text { on } G, \quad u=0 \text { on } \partial G
$$

has a nonnegative solution. It is clear that $w=0$ is a subsolution of (12). The form of $M$ and the standard theorems (cf. e.g. [3]) imply the existence of $z \in W^{2, q}(G)$ such that $M z=1, z=0$ on $\partial G$. Since for the principal eigenfunction $\phi$ of $M$ with 
$\phi=0$ on $\partial G$ we have $\phi>0$ in $G$ and

$$
(M+\gamma) \phi=\left(\lambda_{0}+\gamma\right) \phi>0 \text { in } G,
$$

so by Lemma 1 of [7], $\partial \phi / \partial \nu<0$ on $\partial G$. If $k>0$ is sufficiently large then $v=k \phi+z>0=w$ in $G$ and

$$
M v-\tau v=1+k\left(\lambda_{0}-\tau\right) \phi-\tau z \geqslant 1 \text { in } G .
$$

It follows that $v$ is a supersolution of (12). Therefore by the basic theory (cf. e.g. [1]), there exists $u \in W^{2, q}(G)$ satisfying (12) and $v \geqslant u \geqslant w=0$ in $G$.

Suppose now that $\tau \geqslant \lambda_{0}$ and, contrary to the claim, (12) has a nonnegative solution $u_{1}$. For $\varepsilon>0$ sufficiently small,

$$
M u_{1}-(\tau+\varepsilon) u_{1}=1-\varepsilon u_{1}>0 \text { in } G
$$

and, therefore, $u_{1}$ is a supersolution of the BVP

$$
M u-(\tau+\varepsilon) u=0 \quad \text { in } G, \quad u=0 \quad \text { on } \partial G .
$$

Since

$$
M u_{1}+\gamma u_{1}=1+(\gamma+\tau) u_{1} \geqslant 0 \text { in } G
$$

for any $\gamma>0$, Lemma 1 of [7] implies $u_{1}>0$ in $G$ and $\partial u_{1} / \partial \nu<0$ on $\partial G$. If $\delta>0$ is small then $u_{0} \equiv \delta \phi<u_{1}$ in $G$. Since

$$
M u_{0}-(\tau+\varepsilon) u_{0}=\left(\lambda_{0}-\tau-\varepsilon\right) u_{0} \leqslant 0 \quad \text { in } G,
$$

$u_{0}$ is a subsolution of the BVP (13) with $u_{0}<u_{1}$. This implies the existence of a solution $u$ of the BVP (13) such that $u_{1} \geqslant u \geqslant u_{0}>0$ in $G$. Since this holds for all sufficiently small $\varepsilon>0$ and the eigenvalues of $M$ are isolated we have a contradiction. Q.E.D.

\section{REFERENCES}

1. H. Amann, Fixed point equations and nonlinear eigenvalue problems in ordered Banach spaces, SIAM Rev. 18 (1976), 620-709.

2. J. M. Bony, Principe du maximum dans les espaces de Sobolev, C. R. Acad. Sci. Paris Sér. A 265 (1967), 333-336.

3. M. Chicco, Solvability of the Dirichlet problem in $H^{2, p}(\Omega)$ for a class of linear second order elliptic partial differential equations, Boll. Un. Mat. Ital. 4 (1971), 374-387.

4. M. A. Krasnosel'skii, Positive solutions of operator equations, Noordhoff, Groningen, 1964.

5. P. L. Lions, Problèmes elliptiques de 2 ème ordre non sous forme divergence, Proc. Roy. Soc. Edinburgh Sect. A 84 (1979), 263-271.

6. J. Serrin, A remark on the preceding paper of Amann, Arch. Rational Mech. Anal. 44 (1972), $182-186$.

7. H. Amann and M. G. Crandall, On some existence theorems for semilinear elliptic equations, Indiana Univ. Math. J. 27 (1978), 779-790.

Department of Mathematics, University of Iowa, Iowa City, Iowa 52242 